\title{
Hemobilia: A Rare Complication Following Cholecystectomy
}

Hemobilia is usually attributed to hepatic trauma. Biliary infection, gallstones, aneurysms, and tumors are other rare causes. Hepatic artery aneurysms are rare but mostly fatal lesions. The classical triad of abdominal pain, hemobilia, and jaundice associated with hepatic artery aneurysms is rarely seen, and is usually an autopsy finding (1). The natural course of these aneurysms is to rupture either into the peritoneal cavity or into a surrounding organ such as the biliary tree, producing hemobilia (2). We report here on the case of a patient who presented with hemobilia after cholecystectomy, and underwent successful embolization.

A 45-year-old woman who had undergone an open cholecystectomy at a district hospital ten months previously presented to the emergency room with a 24-hour history of hematemesis and melena. After resuscitation, an upper gastrointestinal endoscopy failed to reveal any abnormality. Initial investigations revealed a serum bilirubin level of $4.8 \mathrm{mg} / \mathrm{dl}$ and an alkaline phosphatase level of $722 \mathrm{IU} / \mathrm{ml}$, with a normal coagulation profile. An ultrasound examination of the abdomen showed that the common bile duct was dilated to $12.8 \mathrm{~mm}$. There was an impression of a mass lesion at the porta hepatis, with an empty gallbladder fossa consistent with previous cholecystectomy. The initial management was conservative, but within 48 hours, rebleeding occurred, and a repeat endoscopy revealed blood emerging from the ampulla of Vater. A contrast CT scan showed a mass at the porta hepatis, suggesting an aneurysm. A celiac axis angiogram demonstrated a pseudoaneurysm of the right hepatic artery (Figure 1) commu- 
nicating with the common bile duct (Figure 2). The right hepatic artery was embolized successfully using a metallic coil (Occluding Spring Emboli, Cook, Inc.). After embolization, the patient made a rapid and uneventful recovery. Liver function tests monitored serially after the embolization remained normal, and the serum bilirubin and alkaline phosphatase levels gradually returned to normal. She was discharged four days after the embolization, and was well at follow-up two months later.

Traumatic pseudoaneurysms of the hepatic artery leading to hemobilia have been reported in the literature (3), but these mainly involve either blunt or penetrating hepatic injury, or are a consequence of a liver biopsy. Up to $80 \%$ of hepatic artery aneurysms involve the extrahepatic course of the vessel, while about $65 \%$ are found in the common hepatic artery (2). In this case, we suspect that trauma to the right hepatic artery during cholecystectomy led to the formation of a pseudoaneurysm, which ruptured into the biliary tract to cause the hemobilia. Jaundice was caused by biliary compression due to the aneurysm, or resulted from the blood clot in the bile duct, or both. Visualization of blood emerging from the ampulla should confirm the diagnosis. In patients presenting with obscure gastrointestinal bleeding, an effort should be made to visualize the ampulla of Vater. If this is not seen and suspicion remains in spite of repeat endoscopy, a diagnostic celiac artery angiogram should be considered.

Embolization of the hepatic artery or the branch feeding the pseudoaneurysm is the treatment of choice, with considerably less morbidity in comparison with surgical ligation (4). However, surgery becomes essential when nonoperative attempts fail to arrest the bleeding, and it is required in cases of tumor or parasitic infection (5). This diagnosis must be considered along with other more common causes of gastrointestinal hemorrhage in patients who have undergone cholecystectomy.

\section{A. M. Jafarey, M. N. Siddiqui, S. Zakaria}

Dept. of Surgery, Aga Khan University Hospital, Karachi, Pakistan

\section{References}

1. Stanley JC, Wakefield TW, Graham IG, et al. Clinical importance and management of splanchnic artery aneurysms. J Vasc Surg 1986: 3: 836-40.

2. Boontje AII. Multiple aneurysms of the visceral branches of the abdominal aorta. Vasa $1979 ; 8: 42-50$.

3. Croce MA, Fabin TC. Traumatic hepatic artery pseudoaneurysm with hemobilia. Am J Surg 1994; 168: 235-8.

4. Lygidakis NJ, Okazaki MM. Iatrogenic hemobilia: how to approach it. Hepatogastroenterology. 1991; 38: 454-7.

5. Bloechie C, Izbicki JR. Hemobilia: presentation, diagnosis and management. Am J Gastroenterol 1994; 89: 1537-40.

\section{Corresponding Author}

M. N. Siddiqui, M.B.Ch.B., F.R.C.S.

University Dept. of Surgery

Faculty of Medicine and Health Sciences

United Arab Emirates University

P.O. Box 17666

Al Ain

United Arab Emirates

Fax: +971-3-678854

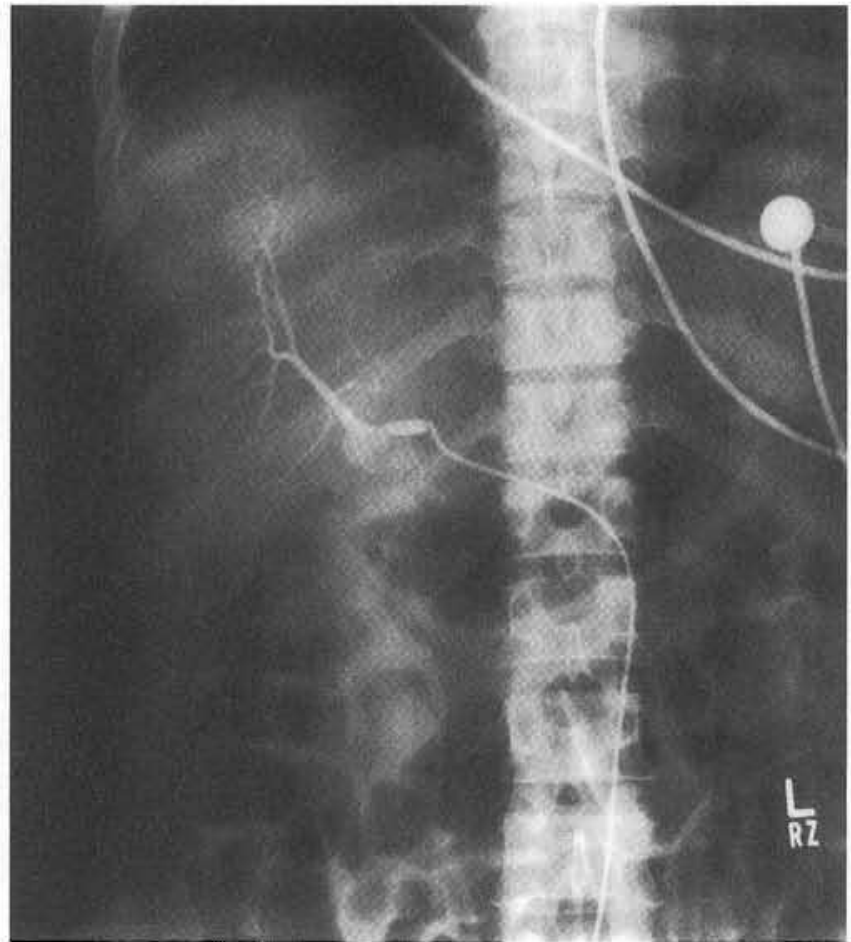

Figure 1: Angiogram of the hepatic artery, showing a pseudoaneurysm.

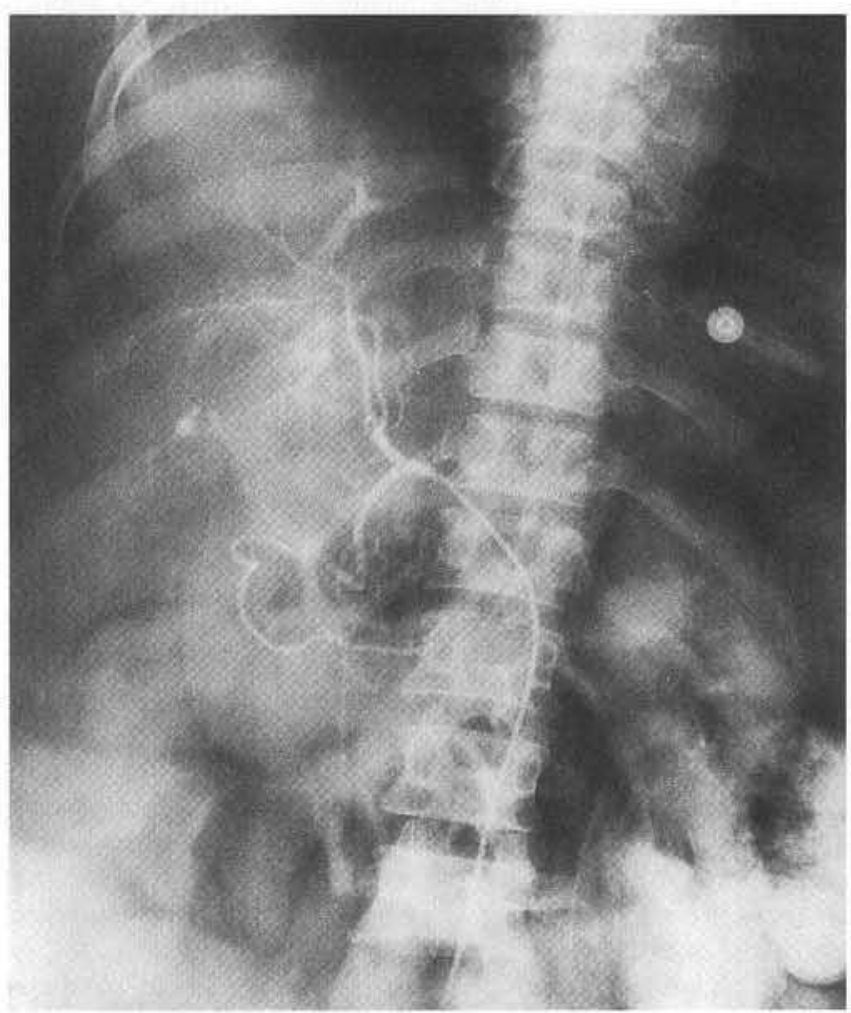

Figure 2: Contrast is seen entering the common bile duct. 